\title{
Centro-Equiaffine Differential Invariants of Curve Families
}

\author{
Yasemin SAĞIROǦLU
}

(Communicated by Murat TOSUN)

\begin{abstract}
The generator set of all centro-equiaffine differential invariant rational functions field for arbitrary curves is obtained. By using these generators, the conditions of equivalence for two curve families are found. Then the relations between elements of generator set are investigated.
\end{abstract}

Keywords: Differential invariant, parametric curve, equivalence.

AMS Subject Classification (2010): 53A35; 53A55.

\section{Introduction}

Invariant theory has been studied for along time on the theory of curves and surfaces. There are many papers on the invariant theory of curves in differential geometry. Also, there are many books on affine differential geometry [12], [18], [20]. In most of the studies, special invariants were considered such as arc length, curvature and torsion. The problem of equivalence has also been investigated.

The concept of affine geometry was introduced by Felix Klein in Erlangen Programme in 1872. According to this programme, affine geometry deals with the properties of curves and surfaces which are invariant under affine maps. Since that time, affine invariants of curves have been investigated. This paper is concerned with the basic theory of centro-equiaffine geometry of curves and related questions of centro-equiaffine invariants. We give the complete system of centro-equiaffine invariants for arbitrary $r$ curves.

In [8] the problem of equivalence investigated for equiaffine curves and [14] it is solved for centro-affine curves. The first comprehensive treatment of affine geometry is given in the seminal work of Paukowitsch [13]. For further developments of subject, we refer the reader to [7], and more modern texts [1], [15], the commentaries [5], [10], [19] and survey papers [11], [3]. The fundamental theorem of curves in centro-affine geometry is obtained in [2]. A discussion of centro-affine plane and space curves can be found in [17]. A detailed discussion of curves in centro-affine geometry can be obtained in [2]. In [6] equiaffine invariants of 3 -dimensional curves and in [4], [13] equiaffine curvatures of $n$-dimensional curves are investigated. Complete systems of global equiaffine invariants for space paths are obtained in [8]. The global $S L(n)$-equivalence of path in $\mathbb{R}^{n}$ is considered in [7] and [16].

The problem of equivalence has been already solved for a single curve and for two curves by Sağıroğlu for the group $S L(n, \mathbb{R})$ [16]. It is solved the equivalence problem for arbitrary $r$ curves in this paper. Firstly, the generator system of $S L(n, \mathbb{R})$-differential invariants for arbitrary $r$ parametric curves is obtained. Then it is given the conditions of equivalence of curve families in terms of generator invariants. It is observed that the generator invariants obtained are functionally independent, namely the generator invariant set is minimal.

Let $\mathbb{R}$ be the field of real numbers and $\mathbb{R}^{n}$ be n-dimensional Euclidean space. The set $S L(n, \mathbb{R})=\{A=$ $\left[a_{i j}\right] \mid i, j=1,2, \ldots, n$ and $a_{i j} \in \mathbb{R}$, which $\left.\operatorname{det} A=1\right\}$ is a group in according to multiplication of matrix. The action of group $S L(n, \mathbb{R})$ on $\mathbb{R}^{n}$ is given by 


$$
g \cdot x=\left(\begin{array}{ccc}
g_{11} & \ldots & g_{1 n} \\
\ldots & \ldots & \ldots \\
g_{n 1} & \ldots & g_{n n}
\end{array}\right) \cdot\left(\begin{array}{c}
x_{1} \\
\vdots \\
x_{n}
\end{array}\right)=\left(\begin{array}{c}
g_{11} x_{1}+\ldots+g_{1 n} x_{n} \\
\vdots \\
g_{n 1} x_{1}+\ldots+g_{n n} x_{n}
\end{array}\right)
$$

for $g \in S L(n, \mathbb{R})$ and $x \in \mathbb{R}$.

Definition 1.1. A $C^{\infty}$-function $x: I \rightarrow \mathbb{R}^{n}$ will be called a parametric curve or briefly a curve in $\mathbb{R}^{n}$.

Definition 1.2. Let $\left\{x_{1}, x_{2}, \ldots, x_{r}\right\}$ and $\left\{y_{1}, y_{2}, \ldots, y_{r}\right\}$ be two pairs of curve families. If $y_{i}=g x_{i}, i=1,2, \ldots, r$ for some $g \in S L(n, \mathbb{R})$, then these curve families will be called $S L(n, \mathbb{R})$-equivalent and denoted by $\left\{x_{1}, x_{2}, \ldots, x_{r}\right\}_{\approx}^{G}\left\{y_{1}, y_{2}, \ldots, y_{r}\right\}$ for the group $G=S L(n, \mathbb{R})$.

Definition 1.3. Let $\left\{x_{1}, x_{2}, \ldots, x_{r}\right\}$ be a curve family in $\mathbb{R}^{n}$. The polynomial

$$
P\left(x_{1}, x_{2}, \ldots, x_{r}\right)=P\left(x_{1}, x_{2}, \ldots, x_{r}, x_{1}^{\prime}, x_{2}^{\prime}, \ldots, x_{r}^{\prime}, \ldots, x_{1}^{(m)}, x_{2}^{(m)}, \ldots, x_{r}^{(m)}\right)
$$

for some natural number $m$ will be called the differential polynomial of $x_{1}, x_{2}, \ldots, x_{r}$.

The derivation of $P\left(x_{1}, x_{2}, \ldots, x_{r}\right)$ will be denoted by $P^{\prime}$ and this derivation is obtained as follows:

$$
x_{\tau}^{(0)}=x_{\tau},\left(x_{\tau}^{(m-1)}\right)^{\prime}=x_{\tau}^{(m)}, \tau=1,2, \ldots, r .
$$

Definition 1.4. Let $P_{1}$ and $P_{2}$ be two differential polynomials. Then the function

$$
f<x_{1}, x_{2}, \ldots, x_{r}>=\frac{P_{1}\left(x_{1}, x_{2}, \ldots, x_{r}\right)}{P_{2}\left(x_{1}, x_{2}, \ldots, x_{r}\right)}, P_{2}\left(x_{1}, x_{2}, \ldots, x_{r}\right) \neq 0
$$

will be called a differential rational function. If

$$
f<g x_{1}, g x_{2}, \ldots, g x_{r}>=f<x_{1}, x_{2}, \ldots, x_{r}>
$$

for some $g \in S L(n, \mathbb{R})$, the differential rational function $f$ is called centro-equiaffine invariant differential rational function. Centro-equiaffine differential polynomial is defined by the same way.

There no exists centro-equiaffine differential polynomial except constant. But there exists the centroequiaffine differential rational function different from constant.

Remark 1.1. Let $\left\{x_{1}, x_{2}, \ldots, x_{r}\right\}_{\approx}^{G}\left\{y_{1}, y_{2}, \ldots, y_{r}\right\}$. So for some $g \in S L(n, \mathbb{R})$ we get $y_{i}=g x_{i}, i=1,2, \ldots, r$. Then for all differential invariant rational function $f$, since

$$
f<y_{1}, y_{2}, \ldots, y_{r}>=f<g x_{1}, g x_{2}, \ldots, g x_{r}>=f<x_{1}, x_{2}, \ldots, x_{r}>
$$

we obtain $f<y_{1}, y_{2}, \ldots, y_{r}>=f<x_{1}, x_{2}, \ldots, x_{r}>$. But the reverse is not true.

The set of all differential rational functions will be denoted by $R<x_{1}, x_{2}, \ldots, x_{r}>$. It is a differential field and $\mathbb{R}$-algebra. Let $G$ be the group $S L(n, \mathbb{R})$. The set of all centro-equiaffine invariant differential rational functions will be denoted by $\left.\left.R<x_{1}, x_{2}, \ldots, x_{r}\right\rangle^{G} . R<x_{1}, x_{2}, \ldots, x_{r}\right\rangle^{G}$ is a differential subfield and subalgebra of $R<x_{1}, x_{2}, \ldots, x_{r}>$.

Definition 1.5. Let $f_{1}, f_{2}, \ldots, f_{k} \in R<x_{1}, x_{2}, \ldots, x_{r}>^{G}$. If the differential field and algebra generated by these functions is equal to $R<x_{1}, x_{2}, \ldots, x_{r}>^{G}$ then these functions will be called the generator set of $R<x_{1}, x_{2}, \ldots, x_{r}>^{G}$.

\section{Centro-Equiaffine Invariants of Arbitrary Curves}

Let $x_{1}, x_{2}, \ldots, x_{n} \in \mathbb{R}^{n}$. The determinant $\left|\begin{array}{ccc}x_{11} & \ldots & x_{n 1} \\ \ldots & \ldots & \ldots \\ x_{1 n} & \ldots & x_{n n}\end{array}\right|$ will be denoted by $\left[x_{1} \ldots x_{n}\right]$. In here, $k$.column of this determinant is consist of the components of $x_{k}$, which are $x_{k 1}, x_{k 2}, \ldots, x_{k n}$.

Lemma 2.1. Let $x_{0}, x_{1}, \ldots, x_{n}, y_{2}, \ldots, y_{n}$ be vectors in $\mathbb{R}^{n}$. Then the following equality holds:

$$
\left[x_{1} x_{2} \ldots x_{n}\right]\left[x_{0} y_{2} \ldots y_{n}\right]-\left[x_{0} x_{2} \ldots x_{n}\right]\left[x_{1} y_{2} \ldots y_{n}\right]-\ldots-\left[x_{1} x_{2} \ldots x_{0}\right]\left[x_{n} y_{2} \ldots y_{n}\right]=0
$$


Proof. [7].

Definition 2.1. A curve $x$ in $\mathbb{R}^{n}$ will be called $S L(n, \mathbb{R})$-regular (briefly regular) if $\left[x x^{\prime} \ldots x^{(n-1)}\right] \neq 0$. Hence for all $t \in I,\left[x(t) x^{\prime}(t) \ldots x^{(n-1)}(t)\right] \neq 0$.

Let $G$ be the group $S L(n, \mathbb{R})$.

Theorem 2.1. Let $x_{1}, x_{2}, \ldots, x_{r}$ be a curve family in $\mathbb{R}^{n}$ such that $x_{1}$ is regular. Then the generator set of $R<x_{1}, x_{2}, \ldots, x_{r}>^{G}$ is

$$
\begin{aligned}
& {\left[x_{1} x_{1}{ }^{\prime} \ldots x_{1}{ }^{(n-1)}\right],\left[x_{1} x_{1}{ }^{\prime} \ldots x_{1}{ }^{(i-1)} x_{1}{ }^{(n)} x_{1}{ }^{(i+1)} \ldots x_{1}{ }^{(n-1)}\right], \quad i=0,1, \ldots, n-1} \\
& {\left[x_{1} x_{1}{ }^{\prime} \ldots x_{1}{ }^{\left({ }^{2}-1\right)} x_{K} x_{1}{ }^{(i+1)} \ldots x_{1}{ }^{(n-1)}\right], \quad i=0,1, \ldots, n-1, K=2,3, \ldots, r .}
\end{aligned}
$$

Proof. For the group $G=S L(n, \mathbb{R})$, the generator set of $R<x_{1}, x_{2}, \ldots, x_{r}>^{G}$ is

$$
\left[x_{1} \ldots x_{n}\right],\left[x_{1} \ldots x_{i-1} x_{\tau} x_{i+1} \ldots x_{n}\right], i=1,2, \ldots, n, \tau \in \Delta /\{1, \ldots, n\}
$$

where $\Delta$ is an index set [20]. Let us take $x_{1}, x_{2}, \ldots, x_{r}, x_{1}^{\prime}, x_{2}^{\prime}, \ldots, x_{r}^{\prime}, x_{1}^{(K)}, x_{2}^{(K)}, \ldots, x_{r}^{(K)}, \ldots$ instead of the vectors $x_{\tau}$. Then the generator set of $\left.R<x_{1}, x_{2}, \ldots, x_{r}, x_{1}^{\prime}, x_{2}^{\prime}, \ldots, x_{r}^{\prime}, x_{1}^{(K)}, x_{2}^{(K)}, \ldots, x_{r}^{(K)}, \ldots>^{G}=R<U\right\rangle^{G}$ is

$$
\begin{gathered}
{\left[x_{1} x_{1}{ }^{\prime} \ldots x_{1}{ }^{(n-1)}\right],\left[x_{1} x_{1}{ }^{\prime} \ldots x_{1}{ }^{(i-1)} x_{1}{ }^{(s)} x_{1}{ }^{(i+1)} \ldots x_{1}{ }^{(n-1)}\right], s \geq n} \\
{\left[x_{1} x_{1}{ }^{\prime} \ldots x_{1}{ }^{(i-1)} x_{K}{ }^{(\tau)} x_{1}{ }^{(i+1)} \ldots x_{1}{ }^{(n-1)}\right], \tau \geq 0, K=2,3, \ldots, r .}
\end{gathered}
$$

We know that $\left[x_{1} x_{1}{ }^{\prime} \ldots x_{1}{ }^{(n-1)}\right]^{\prime}=\left[x_{1} \ldots x_{1}{ }^{(n-2)} x_{1}{ }^{(n)}\right]$.

Firstly, we want to show that $\left[x_{1} x_{1}{ }^{\prime} \ldots x_{1}{ }^{(i-1)} x_{1}{ }^{(s)} x_{1}{ }^{(i+1)} \ldots x_{1}{ }^{(n-1)}\right], s \geq n$ is generated by $\left[x_{1} x_{1}{ }^{\prime} \ldots x_{1}{ }^{(i-1)} x_{1}{ }^{(n)} x_{1}{ }^{(i+1)} \ldots x_{1}{ }^{(n-1)}\right], i=0,1, \ldots, n-2$. Let $s=n$. Then $\left.R<U\right\rangle^{G}$ is generated by (2.2).

Let $s>n$. By induction hypothesis, for $s-1$ let the set (2.2) be the generator set. Therefore $\left[x_{1} x_{1}{ }^{\prime} \ldots x_{1}{ }^{(i-1)} x_{1}{ }^{(s-1)} x_{1}{ }^{(i+1)} \ldots x_{1}{ }^{(n-1)}\right]$ is generated by (2.2). We get

$$
\begin{gathered}
{\left[x_{1} \ldots x_{1}{ }^{(i-1)} x_{1}{ }^{(s)} x_{1}{ }^{(i+1)} \ldots x_{1}{ }^{(n-1)}\right]=\left[x_{1} \ldots x_{1}{ }^{(i-1)} x_{1}{ }^{(s-1)} x_{1}{ }^{(i+1)} \ldots x_{1}{ }^{(n-1)}\right]^{\prime}-} \\
{\left[x_{1} \ldots x_{1}{ }^{(i-2)} x_{1}{ }^{(i)} x_{1}{ }^{\left({ }^{\prime}-1\right)} x_{1}{ }^{(i+1)} \ldots x_{1}{ }^{(n-1)}\right]-\left[x_{1} \ldots x_{1}{ }^{(i-1)} x_{1}{ }^{\left({ }^{(}-1\right)} x_{1}{ }^{(i+1)} \ldots x_{1}{ }^{(n-2)} x_{1}{ }^{(n)}\right] .}
\end{gathered}
$$

In this equality, except of the last determinant, the others is generated by the set (2.2) and in according to induction hypothesis. In Lemma 2.1, if we take $x_{1}=x_{1}, x_{2}=x_{1}^{\prime}, \ldots, x_{n}=x_{1}^{(n-1)}, x_{0}=x_{1}{ }^{(n)}, y_{2}=x_{1}, \ldots$, $y_{i+1}=x_{1}{ }^{(i-1)}, y_{i+2}=x_{1}{ }^{(s-1)}, y_{i+3}=x_{1}{ }^{(i+1)}, \ldots, y_{n}=x_{1}{ }^{(n-2)}$ and eliminate the zero terms it is obtained that

$$
\begin{gathered}
{\left[x_{1} x_{1}{ }^{\prime} \ldots x_{1}{ }^{(n-1)}\right] \cdot\left[x_{1}{ }^{(n)} x_{1} x_{1}{ }^{\prime} \ldots x_{1}{ }^{(i-1)} x_{1}{ }^{(s-1)} x_{1}{ }^{(i+1)} \ldots x_{1}{ }^{(n-2)}\right]+} \\
{\left[x_{1} \ldots x_{1}{ }^{(i-1)} x_{1}{ }^{(n)} x_{1}{ }^{(i+1)} \ldots x_{1}{ }^{(n-1)}\right] \cdot\left[x_{1}{ }^{(i)} x_{1} \ldots x_{1}{ }^{(i-1)} x_{1}{ }^{\left({ }^{(s-1)}\right.} x_{1}{ }^{(i+1)} \ldots x_{1}{ }^{(n-2)}\right]+} \\
{\left[x_{1} \ldots x_{1}{ }^{(n-2)} x_{1}{ }^{(n)}\right] \cdot\left[x_{1}{ }^{(n-1)} x_{1} \ldots x_{1}{ }^{(n-2)}\right]=0 .}
\end{gathered}
$$

So the term $\left[x_{1}{ }^{(n)} x_{1} x_{1}{ }^{\prime} \ldots x_{1}{ }^{(i-1)} x_{1}{ }^{(s-1)} x_{1}{ }^{(i+1)} \ldots x_{1}{ }^{(n-2)}\right]$ generated by the set (2.2).

Similarly, $\left[x_{1} \ldots x_{1}{ }^{(i-1)} x_{K}{ }^{(\tau)} x_{1}{ }^{(i+1)} \ldots x_{1}{ }^{(n-1)}\right], \tau \geq 0, K=2,3, \ldots, r$ is obtained by induction on $\tau$. For $\tau=0$, $\left[x_{1} \ldots x_{1}{ }^{(i-1)} x_{K} x_{1}{ }^{(i+1)} \ldots x_{1}{ }^{(n-1)}\right]$ is the generator. For $\tau=n-1$, let $\left[x_{1} \ldots x_{1}{ }^{(i-1)} x_{K}{ }^{(n-1)} x_{1}{ }^{(i+1)} \ldots x_{1}{ }^{(n-1)}\right]$ generated by the set (2.2) in according to induction hypothesis. Let us show that this is true for $\tau=n$.

$$
\begin{aligned}
& {\left[x_{1} \ldots x_{1}{ }^{(i-1)} x_{K}{ }^{(n-1)} x_{1}{ }^{(i+1)} \ldots x_{1}{ }^{(n-1)}\right]^{\prime}=} \\
& \begin{array}{c}
{\left[x_{1} \ldots x_{1}{ }^{(i-2)} x_{1}{ }^{(i)} x_{K}{ }^{(n-1)} x_{1}{ }^{(i+1)} \ldots x_{1}{ }^{(n-1)}\right]+} \\
{\left[x_{1} \ldots x_{1}{ }^{(i-1)} x_{K}{ }^{(n)} x_{1}{ }^{(i+1)} \ldots x_{1}{ }^{(n-1)}\right]+} \\
{\left[x_{1} \ldots x_{1}{ }^{(i-1)} x_{K}{ }^{(n-1)} x_{1}{ }^{(i+1)} \ldots x_{1}{ }^{(n-2)} x_{1}{ }^{(n)}\right] .}
\end{array}
\end{aligned}
$$

In (2.3), we want to show that the determinant $\left[x_{1} \ldots x_{1}{ }^{(i-1)} x_{K}{ }^{(n)} x_{1}{ }^{(i+1)} \ldots x_{1}{ }^{(n-1)}\right]$ is generated by the set 2.2. Except the determinant

$$
\left[x_{1} \ldots x_{1}{ }^{(i-1)} x_{K}{ }^{(n-1)} x_{1}{ }^{(i+1)} \ldots x_{1}{ }^{(n-2)} x_{1}{ }^{(n)}\right]
$$

other determinants in (2.3) are generated by the set (2.2) and the induction hypothesis. For the last determinant, we use Lemma 2.1. If we take $x_{1}=x_{1}, x_{2}=x_{1}^{\prime}, \ldots, x_{n}=x_{1}{ }^{(n-1)}, x_{0}=x_{1}{ }^{(n)}, y_{2}=x_{1}, \ldots, y_{i+1}=x_{1}{ }^{(i-1)}, y_{i+2}=$ $x_{K}^{(n-1)}, y_{i+3}=x_{1}^{(i+1)}, \ldots, y_{n}=x_{1}^{(n-2)}$ and eliminate the zero terms, it is obtained that 


$$
\begin{gathered}
{\left[x_{1} x_{1}{ }^{\prime} \ldots x_{1}{ }^{(n-1)}\right] \cdot\left[x_{1}{ }^{(n)} x_{1} x_{1}{ }^{\prime} \ldots x_{1}{ }^{(i-1)} x_{K}{ }^{(n-1)} x_{1}{ }^{(i+1)} \ldots x_{1}{ }^{(n-2)}\right]+} \\
{\left[x_{1} \ldots x_{1}{ }^{(i-1)} x_{1}{ }^{(n)} x_{1}{ }^{(i+1)} \ldots x_{1}{ }^{(n-1)}\right] \cdot\left[x_{1}{ }^{(i)} x_{1} \ldots x_{1}{ }^{(i-1)} x_{K}{ }^{(n-1)} x_{1}{ }^{(i+1)} \ldots x_{1}{ }^{(n-2)}\right]+} \\
{\left[x_{1} \ldots x_{1}{ }^{(n-2)} x_{1}{ }^{(n)}\right] \cdot\left[x_{1}{ }^{(n-1)} x_{1} \ldots x_{1}{ }^{(i-1)} x_{K}{ }^{(n-1)} x_{1}{ }^{(i+1)} \ldots x_{1}{ }^{(n-2)}\right]=0 .}
\end{gathered}
$$

So the term $\left[x_{1} \ldots x_{1}{ }^{(i-1)} x_{K}{ }^{(n-1)} x_{1}{ }^{(i+1)} \ldots x_{1}{ }^{(n-2)} x_{1}{ }^{(n)}\right]$ generated by the set $(2.2)$. By the induction hypothesis, the set (2.2) is generator set of $R<U>^{G}$.

Theorem 2.2. Let $G=S L(n, \mathbb{R})$ and $\left\{x_{1}, x_{2}, \ldots, x_{r}\right\}$ and $\left\{y_{1}, y_{2}, \ldots, y_{r}\right\}$ be two curve families such that $x_{1}$ and $y_{1}$ are regular. If for $i=0,1, \ldots, n-1$ and $K=2,3, \ldots, r$

$$
\begin{aligned}
{\left[x_{1} x_{1}{ }^{\prime} \ldots x_{1}{ }^{(n-1)}\right] } & =\left[y_{1} y_{1}{ }^{\prime} \ldots y_{1}{ }^{(n-1)}\right] \\
{\left[x_{1} x_{1}{ }^{\prime} \ldots x_{1}{ }^{(i-1)} x_{1}{ }^{(n)} x_{1}{ }^{(i+1)} \ldots x_{1}{ }^{(n-1)}\right] } & =\left[y_{1} y_{1}{ }^{\prime} \ldots y_{1}{ }^{(i-1)} y_{1}{ }^{(n)} y_{1}{ }^{(i+1)} \ldots y_{1}{ }^{(n-1)}\right] \\
{\left[x_{1} x_{1}{ }^{\prime} \ldots x_{1}{ }^{(i-1)} x_{K} x_{1}{ }^{(i+1)} \ldots x_{1}{ }^{(n-1)}\right] } & =\left[y_{1} y_{1}{ }^{\prime} \ldots y_{1}{ }^{(i-1)} y_{K} y_{1}{ }^{(i+1)} \ldots y_{1}{ }^{(n-1)}\right]
\end{aligned}
$$

then $\left\{x_{1}, x_{2}, \ldots, x_{r}\right\}_{\approx}^{G}\left\{y_{1}, y_{2}, \ldots, y_{r}\right\}$.

Proof. Since $x_{1}$ and $y_{1}$ are regular, we get $\left[x_{1} x_{1}{ }^{\prime} \ldots x_{1}{ }^{(n-1)}\right] \neq 0$ and $\left[y_{1} y_{1}{ }^{\prime} \ldots y_{1}{ }^{(n-1)}\right] \neq 0$. Let us take the matrices

$$
A_{x_{1}}=\left(\begin{array}{ccc}
x_{11}(t) & \ldots & x_{11}{ }^{(n-1)}(t) \\
\ldots & \ldots & \ldots \\
x_{1 n}(t) & \ldots & x_{1 n}^{(n-1)}(t)
\end{array}\right) \text { and } A_{x_{1}}^{\prime}=\left(\begin{array}{ccc}
x_{11}{ }^{\prime}(t) & \ldots & x_{11}{ }^{(n)}(t) \\
\ldots & \ldots & \ldots \\
x_{1 n^{\prime}(t)} & \ldots & x_{1 n}{ }^{(n)}(t)
\end{array}\right)
$$

Since $\left[x_{1} x_{1}{ }^{\prime} \ldots x_{1}{ }^{(n-1)}\right] \neq 0$, there exists matrix inverse of $A_{x_{1}}$. Take the matrix $A_{x_{1}}^{-1} \cdot A_{x_{1}}^{\prime}=C$. Then $A_{x_{1}}^{\prime}=A_{x_{1}} \cdot C$. So the matrix $C$ has the form

$$
C=\left(\begin{array}{cccc}
0 & \ldots & 0 & c_{1 n} \\
1 & \ldots & 0 & c_{2 n} \\
\ldots & \ldots & \ldots & \ldots \\
0 & \ldots & 1 & c_{n n}
\end{array}\right)
$$

where

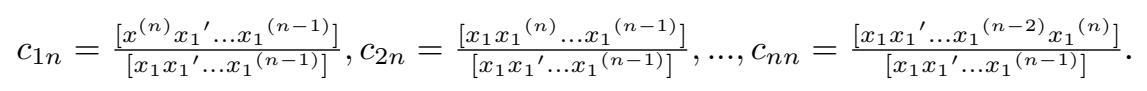

From conditions of the theorem, it is obtained that $A_{x_{1}}^{-1} \cdot A_{x_{1}}^{\prime}=A_{y_{1}}^{-1} \cdot A_{y_{1}}^{\prime}$. So we have that

$$
\begin{gathered}
\left(A_{y_{1}} \cdot A_{x_{1}}^{-1}\right)^{\prime}=A_{y_{1}}^{\prime} \cdot A_{x_{1}}^{-1}+A_{y_{1}} \cdot\left(A_{x_{1}}^{-1}\right)^{\prime}=A_{y_{1}}^{\prime} \cdot A_{x_{1}}^{-1}+A_{y_{1}} \cdot\left(-A_{x_{1}}^{-1} \cdot A_{x_{1}}^{\prime} \cdot A_{x_{1}}^{-1}\right) \\
=A_{y_{1}} \cdot\left(A_{y_{1}}^{-1} \cdot A_{y_{1}}^{\prime}-A_{x_{1}}^{-1} \cdot A_{x_{1}}^{\prime}\right) \cdot A_{x_{1}}^{-1}=0
\end{gathered}
$$

Therefore $A_{y_{1}} \cdot A_{x_{1}}^{-1}=g, g$ is constant. And we get $A_{y_{1}}=g A_{x_{1}}$. So $\operatorname{det} A_{y_{1}}=\operatorname{det}\left(g A_{x_{1}}\right)$ and since $\left[x_{1} x_{1}{ }^{\prime} \ldots x_{1}{ }^{(n-1)}\right]=\left[y_{1} y_{1}{ }^{\prime} \ldots y_{1}{ }^{(n-1)}\right]$, then it is obtain that $g \in S L(n, \mathbb{R})$. If we write this equality obviously, we have that $y_{1}(t)=g x_{1}(t), \forall t \in I$.

Let us take the matrix

$$
D_{x_{K}}=\left(\begin{array}{cccc}
x_{11}(t) & \ldots & x_{11}{ }^{(n-2)}(t) & x_{K 1}(t) \\
x_{12}(t) & \ldots & x_{12}{ }^{(n-2)}(t) & x_{K 2}(t) \\
\ldots & \ldots & \ldots & \ldots \\
x_{1 n}(t) & \ldots & x_{1 n}{ }^{(n-2)}(t) & x_{K n}(t)
\end{array}\right)
$$

Let take $A_{x_{1}}^{-1} \cdot D_{x_{K}}=H=\left[h_{i j}\right], i, j=1,2, \ldots, n$. Let us find the elements of this matrix. We have that $D_{x_{K}}=$ $A_{x_{1}}$.H. Then similarly we get that

$$
H=\left(\begin{array}{cccc}
1 & \ldots & 0 & h_{1 n} \\
0 & \ldots & 0 & h_{2 n} \\
\ldots & \ldots & \ldots & \ldots \\
0 & \ldots & 0 & h_{n n}
\end{array}\right)
$$

where

$$
h_{1 n}=\frac{\left[x_{K} x_{1}{ }^{\prime} \ldots x_{1}{ }^{(n-1)}\right]}{\left[x_{1} x_{1}{ }^{\prime} \ldots x_{1}{ }^{(n-1)}\right]}, h_{2 n}=\frac{\left[x_{1} x_{K} \ldots x_{1}{ }^{(n-1)}\right]}{\left[x_{1} x_{1}{ }^{\prime} \ldots x_{1}{ }^{(n-1)}\right]}, \ldots, h_{n n}=\frac{\left[x_{2} x_{1}{ }^{\prime} \ldots x_{1}{ }^{(n-2)} x_{K}\right]}{\left[x_{1} x_{1}{ }^{\prime} \ldots x_{1}{ }^{(n-1)}\right]}
$$


Similarly, we can find the matrix $A_{y_{1}}^{-1} \cdot D_{y_{K}}$. From conditions of the theorem, we have that $A_{x_{1}}^{-1} \cdot D_{x_{K}}=$ $A_{y_{1}}^{-1}$. $D_{y_{K}}$. We know that $A_{y_{1}}=g A_{x_{1}}$. Therefore we get

$$
A_{x_{1}}^{-1} \cdot D_{x_{K}}=\left(g A_{x_{1}}\right)^{-1} \cdot D_{y_{K}}=A_{x_{1}}^{-1} \cdot g^{-1} \cdot D_{y_{K}}
$$

and then

$$
D_{x_{K}}=g^{-1} \cdot D_{y_{K}} \Longrightarrow D_{y_{K}}=g \cdot D_{x_{K}} .
$$

Then we get $y_{K}(t)=g x_{K}(t), \forall t \in I, K=2,3, \ldots, r$. So for the same $g \in S L(n, \mathbb{R})$, it is obtained that $y_{1}(t)=g x_{1}(t)$ and $y_{K}(t)=g x_{K}(t)$. Therefore we get $y_{K}(t)=g x_{K}(t), \forall t \in I, K=1,2, \ldots, r$. Hence $\left\{x_{1}, x_{2}, \ldots, x_{r}\right\}_{\approx}^{G}\left\{y_{1}, y_{2}, \ldots, y_{r}\right\}$.

Theorem 2.3. Let $G=S L(n, \mathbb{R})$ and $f_{1}(t), f_{2}(t), \ldots, f_{n}(t), f_{n}(t) \neq 0$ and $f_{K i}(t)(i=1,2, \ldots, n-1, K=2,3, \ldots, r)$ be $C^{\infty}$-functions on $I$. Then there exist curves $x_{1}, x_{2}, \ldots, x_{r}$ where $x_{1}$ is regular such that

$$
\begin{aligned}
{\left[x_{1} \ldots x_{1}{ }^{(i-1)} x_{1}{ }^{(n)} x_{1}{ }^{(i+1)} \ldots x_{1}{ }^{(n-1)}\right] } & =f_{i}(t), i=0,1, \ldots, n-1 \\
{\left[x_{1} x_{1}{ }^{\prime} \ldots x_{1}{ }^{(n-1)}\right] } & =f_{n}(t) \\
{\left[x_{1} \ldots x_{1}{ }^{(i-1)} x_{K} x_{1}^{(i+1)} \ldots x_{1}{ }^{(n-1)}\right] } & =f_{K i}(t), i=0,1, \ldots, n-1, K=2,3, \ldots, r .
\end{aligned}
$$

Proof. Including an $x_{1}$ unknown,

$$
\begin{aligned}
\left.\frac{\left[x_{1} \ldots x_{1}{ }^{(i-1)} x_{1}{ }^{(n)} x_{1}{ }^{(i+1)} \ldots x_{1}{ }^{(n-1)}\right]}{\left[x_{1} x_{1}{ }^{\prime} \ldots x_{1}(n-1)\right.}\right] & =\frac{f_{i}(t)}{f_{n}(t)}=g_{i}(t), i=0,1, \ldots, n-1 \\
\frac{\left[x_{1} \ldots x_{1}{ }^{(i-1)} x_{K} x_{1}{ }^{(i+1)} \ldots x_{1}{ }^{(n-1)}\right]}{\left[x_{1} x_{1}{ }^{\prime} \ldots x_{1}{ }^{(n-1)}\right]} & =\frac{f_{K i}(t)}{f_{n}(t)}=g_{K i}(t), i=0, \ldots, n-1, K=2, \ldots, r .
\end{aligned}
$$

We take the matrix multiplication $A_{x_{1}}^{-1} \cdot A_{x_{1}}^{\prime}=B$ such that $A_{x_{1}}^{\prime}=A_{x_{1}} . B$. In here, matrix $B$ has the form

$$
B=\left(\begin{array}{cccc}
0 & \ldots & 0 & g_{1}(t) \\
1 & \ldots & 0 & g_{2}(t) \\
\ldots & \ldots & \ldots & \ldots \\
0 & \ldots & 1 & g_{n}(t)
\end{array}\right) .
$$

Then we have the following differential equation system from this multiplication:

$$
\begin{gathered}
x_{11} g_{1}(t)+x_{11}{ }^{\prime} g_{2}(t)+\ldots+x_{11}{ }^{(n-1)} g_{n}(t)=x_{11}{ }^{(n)} \\
x_{12} g_{1}(t)+x_{12}{ }^{\prime} g_{2}(t)+\ldots+x_{12}{ }^{(n-1)} g_{n}(t)=x_{12}{ }^{(n)} \\
\vdots \\
x_{1 n} g_{1}(t)+x_{1 n^{\prime}} g_{2}(t)+\ldots+x_{1 n}{ }^{(n-1)} g_{n}(t)=x_{1 n}{ }^{(n)}
\end{gathered}
$$

Let we take $x_{1 i}=y, i=1,2, \ldots, n$. So we can write the above differential equation system as $g_{1}(t) y+g_{2}(t) y^{\prime}+$ $\ldots+g_{n}(t) y^{(n-1)}-y^{(n)}=0$.

It is known that the theory of differential equations, there exist one solution of this differential equation. Let $x_{1}(t)=\left(y_{1}, y_{2}, \ldots, y_{n}\right)$ be the solution. Then the curve $x_{1}(t)$ satisfies the conditions of the theorem.

Similarly, take the matrices $D_{x_{K}}$ and $A_{x_{1}}$. Let $A_{x_{1}}^{-1} \cdot D_{x_{K}}=C$. So $D_{x_{K}}=A_{x_{1}} . C$. Then we get the matrix $C$ as:

$$
C=\left(\begin{array}{ccccc}
1 & 0 & \ldots & 0 & g_{K 0}(t) \\
0 & 1 & \ldots & 0 & g_{K 1}(t) \\
\ldots & \ldots & \ldots & \ldots & \ldots \\
0 & 0 & \ldots & 1 & g_{K n-2}(t) \\
0 & 0 & \ldots & 0 & g_{K n-1}(t)
\end{array}\right) .
$$

Since $D_{x_{K}}=A_{x_{1}} . C$, we have the following differential equation system:

$$
\begin{gathered}
x_{K 1}=x_{11} g_{K 0}(t)+x_{11}{ }^{\prime} g_{K 1}(t)+\ldots+x_{11}{ }^{(n-1)} g_{K n-1}(t) \\
x_{K 2}=x_{12} g_{K 0}(t)+x_{12}{ }^{\prime} g_{K 1}(t)+\ldots+x_{12}{ }^{(n-1)} g_{K n-1}(t) \\
\vdots \\
x_{K n}=x_{1 n} g_{K 0}(t)+x_{1 n^{\prime}} g_{K 1}(t)+\ldots+x_{1 n}{ }^{(n-1)} g_{K n-1}(t)
\end{gathered}
$$


So we get the curves $x_{K}=\left(\begin{array}{c}x_{K 1} \\ x_{K 2} \\ \vdots \\ x_{K n}\end{array}\right), K=2,3, \ldots, r$.

It is obtained that $\frac{\left[x_{1} x_{1}{ }^{\prime} \ldots x_{1}{ }^{(n-1)}\right]^{\prime}}{\left[x_{1} x_{1}{ }^{\prime} \ldots x_{1}{ }^{(n-1)}\right]}=\frac{f_{n}{ }^{\prime}(t)}{f_{n}(t)}$. Let $\left[x_{1} x_{1}{ }^{\prime} \ldots x_{1}{ }^{(n-1)}\right]=p(t)$. Then we get $\frac{p^{\prime}(t)}{p(t)}=\frac{f_{n}{ }^{\prime}(t)}{f_{n}(t)}$.

So we get for some $\lambda \in \mathbb{R} /\{0\}, f_{n}(t)=\lambda p(t)$. Then it can be found a matrix $h$ such that deth $\neq 0$. Let $y(t)$ be the curve $h x_{1}(t)$ and $y_{K}(t)$ be the curves $h x_{K}(t), K=2,3, \ldots, r$. Then $y(t)$ and $y_{K}(t)$ are curves which provide the conditions of the theorem. Really, since

$$
\left[y \ldots y^{(n-1)}\right]=\left[\left(h x_{1}\right) \ldots\left(h x_{1}\right)^{(n-1)}\right]=\left[h x_{1} \ldots h x_{1}^{(n-1)}\right]=\operatorname{deth} \cdot\left[x_{1} \ldots x_{1}^{(n-1)}\right]=f_{n}(t) \neq 0
$$

then $y(t)$ is regular.

$$
\begin{aligned}
& \frac{\left[y \ldots y^{(i-1)} y^{(n)} y^{(i+1)} \ldots y^{(n-1)}\right]}{\left[y \ldots y^{(n-1)}\right]}=\frac{\operatorname{deth} .\left[x_{1} \ldots x_{1}{ }^{(i-1)} x_{1}{ }^{(n)} x_{1}{ }^{(i+1)} \ldots x_{1}{ }^{(n-1)}\right]}{\operatorname{deth} \cdot\left[x_{1} x_{1}{ }^{\prime} \ldots x_{1}^{(n-1)}\right]}=\frac{f_{i}(t)}{f_{n}(t)} \\
& \frac{\left[y \ldots y^{(i-1)} y_{K} y^{(i+1)} \ldots y^{(n-1)}\right]}{\left[y \ldots y^{(n-1)}\right]}=\frac{\operatorname{deth} \cdot\left[x_{1} \ldots x_{1}{ }^{(i-1)} x_{K} x_{1}{ }^{(i+1)} \ldots x_{1}{ }^{(n-1)}\right]}{\operatorname{deth} \cdot\left[x_{1} x_{1}{ }^{\prime} \ldots x_{1}^{(n-1)}\right]}=\frac{f_{K i}(t)}{f_{n}(t)}
\end{aligned}
$$

and since $\left[y \ldots y^{(n-1)}\right]=f_{n}(t)$, then for $i=0,1, \ldots, n-1, K=2,3, \ldots, r$ we get

$$
\begin{aligned}
{\left[y \ldots y^{(i-1)} y^{(n)} y^{(i+1)} \ldots y^{(n-1)}\right] } & =f_{i}(t) \\
{\left[y \ldots y^{(i-1)} y_{K} y^{(i+1)} \ldots y^{(n-1)}\right] } & =f_{K i}(t) .
\end{aligned}
$$

Hence curves $y(t)$ and $y_{K}(t), K=2,3, \ldots, r$ satisfy conditions of the theorem.

\section{References}

[1] Aripov, R.G., Khadziev, D., The Complete System of Global Differential and Integral Invariants of a Curve in Euclidean Geometry, 51 (2007),no.7, 1-14.

[2] Gardner, R.B., Wilkens, G.R., The Fundamental Theorems of Curves and Hypersurfaces in Centro-affine Geometry, Bull.Belg.Math.Soc., 4 (1997), 379-401.

[3] Giblin, J.P., Sapiro, G., Affine-invariant distances, envelopes and symmetry sets, Geometriae Dedicata, 71 (1998), $237-261$.

[4] Guggenheimer, H.W., Differential Geometry, McGraw-Hill, New York, 1963.

[5] Hann C.E., Hickman, M.S., Projective Curvature and Integral Invariants, Acta Applicandae Mathematicae, 74 (2002), 177-193.

[6] Izumiyma, S., Sano, T., Generic affine differential geometry of space curves, Proceedings of the Royal Society of Edinburgh, 128A (1998), 301-314.

[7] Khadjiev, D., The Application of Invariant Theory to Differential Geometry of Curves, Fan Publ., Tashkent, 1988.

[8] Khadjiev, D., Pekşen, Ö., The complete system of global differential and integral invariants for equi-affine curves, Differential Geometry and It's Applications, 20 (2004), 167-175.

[9] Klingenberg W., A Course in Differential Geometry, Springer-Verlag, New York, 1978.

[10] Looijenga, E.J.N., Invariants of quartic plane curves as automorphic forms, Contemporary Mathematics, 422 (2007), 107-120.

[11] Mokhtarian, F., Abbasi, S., Affine Curvature Space Scale with Affine Length Parametrization, Pattern Analysis \& Applications, 4 (2001), $1-8$.

[12] Nomizu, K., Sasaki, T., Affine Differential Geometry, Cambridge Univ. Pres., 1994.

[13] Paukowitsch, H.P., Begleitfiguren und Invariantensystem Minimaler Differentiationsordnung von Kurven im Reellen n-dimensionalen Affinen Raum, Mh. Math., 85 (1978), no.2, 137-148.

[14] Pekşen, Ö., Khadjiev, D., On invariants of curves in centro-affine geometry, J. Math. Kyoto Univ., 44 (2004), no.3, 603-613.

[15] Sağıroğlu, Y., Global differential invariants of affine curves in $\mathbb{R}^{2}$, Far East Journal of Mathematical Sciences, 96 (2015), no.4, $497-515$.

[16] Sağıroğlu, Y., The equivalence of curves in $S L(n, \mathbb{R})$ and its application to ruled surfaces, Appl. Math. Comput., 218 (2011), $1019-1024$.

[17] Schirokow, P.A., Schirokow, A.P., Affine Differential Geometrie, Teubner, Leipzig, 1962.

[18] Su, B., Affine Differential Geometry, Science Press, Beijing, Gordon and Breach, New York, 1983.

[19] Ünel, M., Wolovich, W.A., On the Construction of Complete Sets of Geometric Invariants for Algebraic Curves, Advances in Applied Mathematics, 24 (2000), 65-87.

[20] Weyl, H., The Classical Groups, Princeton Univ. Press, Princeton, NJ, 1946. 


\section{Affiliations}

YASEMIN SAĞIROĞLU

ADDRESS: Karadeniz Technical University, Dept. of Mathematics, 61080, Trabzon-TURKEY.

E-MAIL: sagiroglu.yasemin@gmail.com 